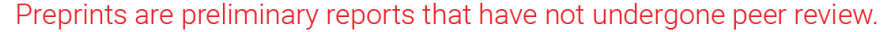 They should not be considered conclusive, used to inform clinical practice, or referenced by the media as validated information. \\ The efficacy of a motorized lower-limb prosthetic device: a pilot study
}

Jo Ghillebert ( $\square$ jo.ghillebert@vub.be)

Vrije Universiteit Brussel https://orcid.org/0000-0002-4716-5393

Joost Geeroms

Vrije Universiteit Brussel

\section{Louis Flynn}

Vrije Universiteit Brussel

\section{Sander De Bock}

Vrije Universiteit Brussel

\section{Renée Govaerts}

Vrije Universiteit Brussel

\section{Simona Crea}

Scuola Superiore Sant'Anna

Nicola Vitiello

Scuola Superiore Sant'Anna

\section{Dirk Lefeber}

Vrije Universiteit Brussel

\section{Bart Roelands}

Vrije Universiteit Brussel

\section{Romain Meeusen}

Vrije Universiteit Brussel

\section{Kevin De Pauw}

Vrije Universiteit Brussel

\section{Research}

Keywords: Transfemoral, Amputation, Powered, Prostheses, Ankle, Knee, Clinical evaluation, Daily activities, Robotics

Posted Date: July 29th, 2020

DOI: https://doi.org/10.21203/rs.2.22808/v2

License: (9) This work is licensed under a Creative Commons Attribution 4.0 International License. 

The authors have withdrawn this preprint from Research Square 